\title{
Effect Analysis of Mega-sub Structure with Isolation Bearing
}

\author{
H.Wu', a, Y.Z.Li' ${ }^{1, b}$, Z.H.Li, ${ }^{1, c}$ \\ ${ }^{1}$ Xi'an University of Technology, School of Civil Engineering and Architecture, Xi'an 710048, China \\ awuhaonwpu@163.com, b506960357@qq.com, clizhihui@xaut.edu.cn
}

Keywords: Mega-sub structure; ANSYS; isolation bearing unit; modal analysis; time-history analysis

Abstract. Mega-sub structure is a new type of structure system of shock absorption. In order to give full play to its shock isolation performance, in this paper we utilize the finite element method software ANSYS to establish isolation bearing units evenly arranged in the bottom of the substructure column, which forms a new Mega-sub shock isolation structure system. Meanwhile, for the sake of analyzing the isolation effect of the Mega-sub structure, a three-dimensional finite element simplification model of Mega-sub structure is established as well; and then Mega-sub structure is compared with the general mega-frame structure. First, the self-vibration period and frequency of the two structures are discussed by the modal analysis. Second, with the time-history analysis of the same seismic wave carried out, we analyze the corresponding values including displacement and acceleration of the two structures. It turns out that Mega-sub structure with isolation bearing possesses a good vibration isolation effect.

\section{Introduction}

Mega-sub controlled structure system springing up as a novel structural system in the ultra-high-rise buildings is a two-level structural system which applies the tuned mass principle of tuned mass dampers (TMD) and Multiple Tuned Mass Damper (MTMD) and takes the subframe in the traditional mega structure as the substructure. The control-response strategy is: after the external load as wind or earthquake etc. to main structure, it transfers the energy to substructure and then the energy dissipates by the mutual tuning of main structure and substructure. Maria Q.Feng [1] simplified main structure and substructure to one degree of freedom respectively, and optimized the parameters by analyzing the interrelation of damping ratio in substructure and mean square value of target response. Zhang [2] simplified main structure and substructure to a system with many degrees of freedom and optimized the parameters by complex mode analysis. Lian [3] found the damping effect is better when they place the damper between the top of substructure and main structure. Li and Zhang [4] investigated the response of mega-sub structure system in the rare earthquake and derived the optimal damping parameter. Based on these researches, this paper will further explore the properties and response regularity of vibration-absorption device in mega-sub structure so that we can provide overall and reliable theoretical data for the structural design of the ultra-high-rise buildings.

\section{The Simulation of Isolation Bearing [5]}

The simulation of isolation bearing is a combination of nonlinear springs in two horizontal directions, viscous damper, and linear springs in vertical direction. We choose the combin14 element to simulate vertical stiffness and combin40 element to horizontal stiffness. The mechanical principle is as in figure 1. 


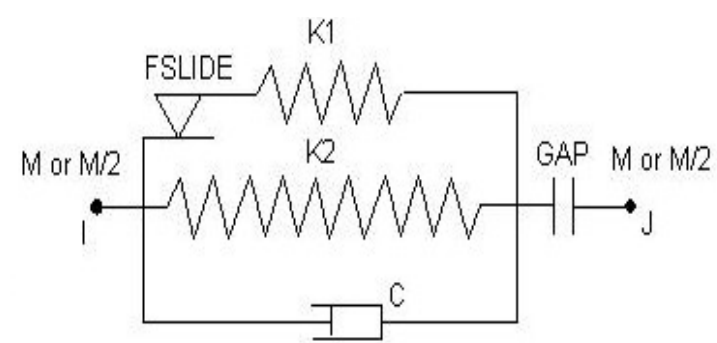

Fig. 1 combin40 mechanics schematic diagram

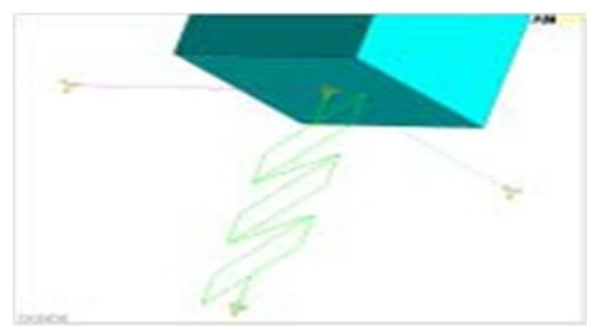

Fig. 2 Isolation bearing unit diagram

\section{The Finite Element Computing Model}

Mega-sub structure consists of 3 mega layers main structure, and each contains 10 layers substructure. The first substructure is the traditional frame structure while the second and third substructure has the capability of frequency adjustment. The story height of main structure is $48 \mathrm{~m}$, it of substructure is $4 \mathrm{~m}$, the plane size is $32 \mathrm{~m} \times 32 \mathrm{~m}$, the cross section of square steel applied in the mega column is $1.5 \mathrm{~m} \times 1.5 \mathrm{~m} \times 0.04 \mathrm{~m}$, and the cross section of I-steel applied in mega beam is $1.5 \mathrm{~m} \times 1.5 \mathrm{~m} \times 0.03 \mathrm{~m}$. The cross section of square steel in the substructure is $0.3 \mathrm{~m} \times 0.3 \mathrm{~m} \times 0.01 \mathrm{~m}$, and it of I-steel is $0.3 \mathrm{~m} \times 0.3 \mathrm{~m} \times 0.008 \mathrm{~m}$. The steel is Q235, of which the elasticity modulus is $2.06 \times 1011 \mathrm{~N} / \mathrm{m} 2$ and Poisson ratio is 0.3 . The centroid of the isolation bearing at the bottom of the substructure coincident with it of the cross section of the column. The isolation bearing is rubber (LRN300-60) bearing, of which the vertical stiffness is $8.96 \times 105 \mathrm{kN} / \mathrm{m}$, horizontal stiffness is $660 \mathrm{kN} / \mathrm{m}$, and the damping ratio is 0.03 . The simplified model by finite element method is as shown in figure 3 .

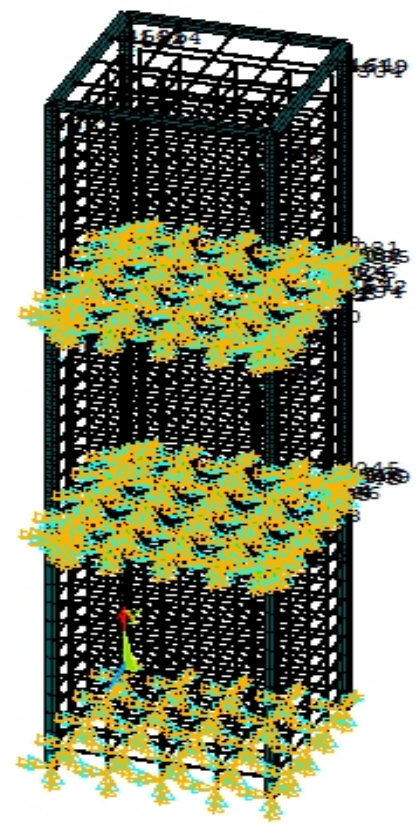

Fig. 3 Simplified model of Mega-sub

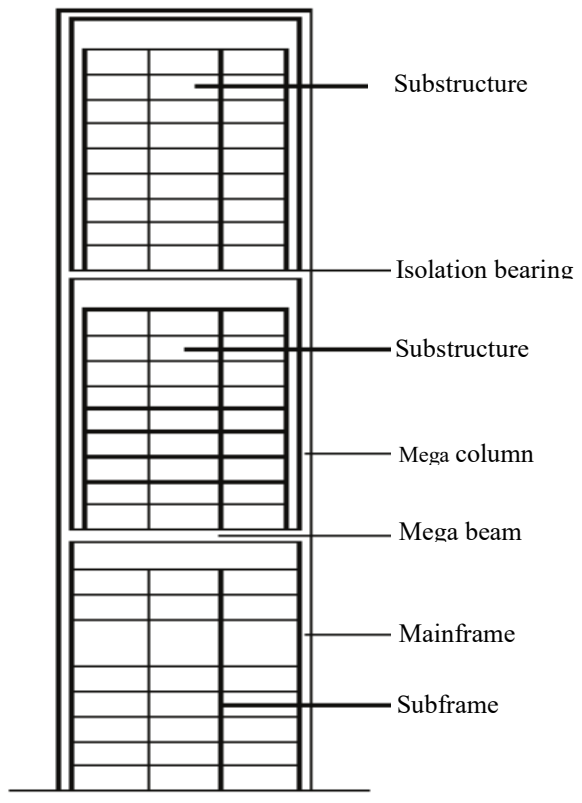

Fig. 4 Mega-sub shock isolation structure

\section{The Time-History Analysis}

\section{The Mode Analysis}

We utilize finite element software ANSYS to compute mode of shock absorption mega-sub structure and traditional mega frame structure, and vibration characteristics of the structure state its superiority.

Table 1 displays the natural vibration frequency at the first 8 steps. The analysis shows that the natural vibration frequency of mega-sub structure is lower than it of the traditional structure. At the first step, the natural vibration frequency of mega-sub structure is $0.25679 \mathrm{~Hz}$, deceasing by $7.75 \%$ than it of the traditional structure, $0.27837 \mathrm{~Hz}$. Meanwhile, the variation in the low mode is more 
obvious than it in high mode, and the rate of periodic convergence in mega-sub structure is faster than it in traditional mega structure.

Table 1 Comparison of the natural vibration frequency at the first 8 steps

\begin{tabular}{c|c|c}
\hline mode of vibration & $\begin{array}{c}\text { Frequency of mega-sub shock } \\
\text { isolation structure }(\mathrm{Hz})\end{array}$ & $\begin{array}{c}\text { Frequency of traditional mega } \\
\text { frame structure }(\mathrm{Hz})\end{array}$ \\
\hline 1 & 0.25679 & 0.27837 \\
3 & 0.25679 & 0.27837 \\
4 & 0.27988 & 0.31363 \\
5 & 0.76083 & 0.87249 \\
6 & 0.76083 & 0.87249 \\
7 & 0.87931 & 0.96982 \\
8 & 0.92038 & 1.4624 \\
& 0.92038 & 1.5041 \\
\hline
\end{tabular}

To sum up, when using the isolation bearing, the period of mega-sub structure increases, and the natural vibration period of buildings prolongs and is far from the characteristic period. Thereby, the mainly frequency band with energy in earthquake is avoided and the response to earthquake of the upper structure is relieved. The frequency and period comparison of the mega-sub shock absorption structure and the traditional mega frame structure is in figure 5.

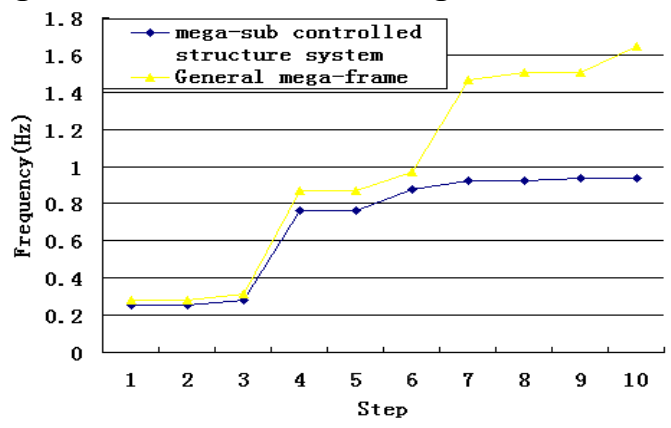

Fig. 5 the frequency comparison at the first 10 steps

\section{The Time-history Analysis}

In this paper the El-Centro seismic wave was chosen to carry on the earthquake time-history analysis. Figure 6 to figure 9 is the displacement and acceleration time-history curve. As shown in the figures, the displacement and acceleration of the top of the main structure in mega-sub shock isolation structure apparently decrease comparing with the top of the traditional mega frame structure. The displacement and acceleration of the top in the buildings are one of the criteria to estimate the safety of high-rise buildings. Thus, mega-sub shock isolation structure can effectively restrict the response and then control its lateral displacement and failure.

The peak value of displacement response is $39.56 \mathrm{~cm}$ in the traditional mega frame structure, while it is $30.76 \mathrm{~cm}$ in the mega-sub shock isolation structure, seismic reduction by $22 \%$. The peak value of acceleration response is $8.443 \mathrm{~m} / \mathrm{s} 2$ in the traditional mega frame structure, while it is $6.320 \mathrm{~m} / \mathrm{s} 2$ in the mega-sub shock isolation structure, seismic reduction by $25 \%$.

\section{Conclusions}

The time-history study of mega-sub structure indicates that the isolation bearing can effectively reduce the value of structural displacement and acceleration, with seismic reduction by $22 \%$ and $25 \%$ respectively, so that the structural lateral displacement and failure can be restricted in the role of earthquake. Therefore, it is practicable to employ the isolation bearing in the mega-sub structure. 


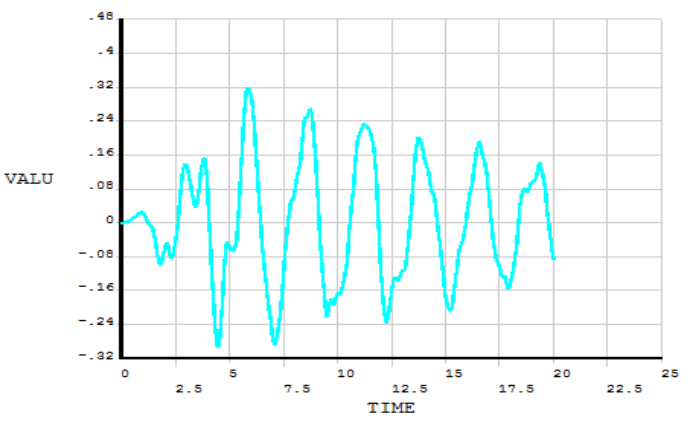

Fig. 6 X-direction displacement curve of the top of the main structure in Mega-sub shock isolation structure

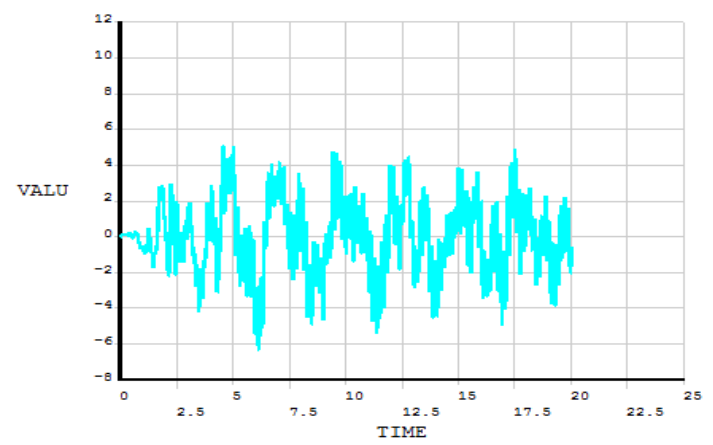

Fig. $8 \mathrm{X}$-direction acceleration time-history curve of the top of the main structure in Mega-sub shock isolation structure

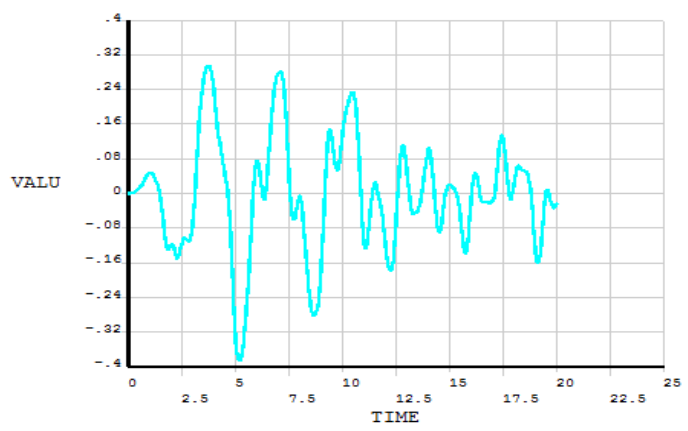

Fig. 7 X-direction displacement curve of the top of the general mega-frame structure

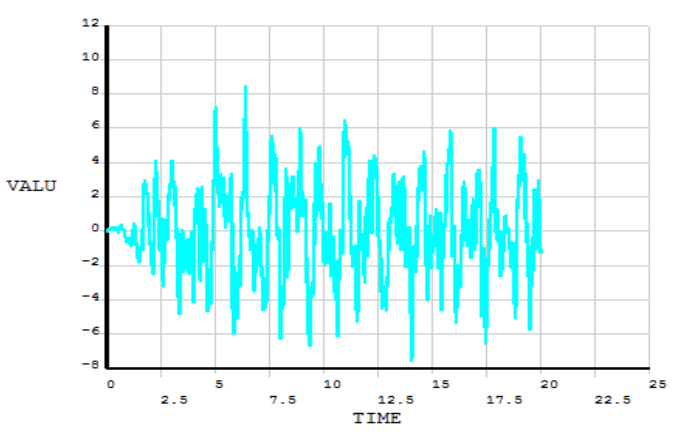

Fig. 9 X-direction acceleration time-history curve of the top of the general mega-frame structure

\section{Acknowledgements}

This work was financially supported by the Special Research Program of Education Department of Shaanxi Provincial(16JK1547), Science and Technology Innovation Project of Xi'an University of Technology(2014CX017) and Natural Science Basic Research Plan in Shaanxi Province (2017JQ5062).

\section{References}

[1] Maria Q.Feng, A.Mita: Vibration Control of Tall Buildings Using Mega-Sub Configuration. Journal of Engineering Mechanics. Vol. 121(1995), p. 1082-1087.

[2] X.A.Zhang, J.L.Zhang, J.S.Jiang: On Improving the Self-Absorption of Shock of Mega-Frame Building. Journal of Northwestern Polytechnical University. Vol. 22(2004), p. 59-63.

[3] Y.D.Lian, X.A.Zhang, Z.X.Wang: Investigation in New Building Structure With Different Mass Ratio Between Mega-Structure and Sub-Structure. Journal of Vibration and Shock. Vol. 26(2007), p. 112-115.

[4] T.Li, X.A.Zhang: Research on parameters of viscous dampers inserted in MSCSS. Earthquake Engineering and EngineeringVibration. Vol. 31(2011), p. 71-76.

[5] W.G.Wang, H.Y.Sheng: A Elasto-Plastic Model of The Rubber Bearings Base-Isolation System. Journal of He Fei University of Technology. Vol. 29(2006), p. 193-196. 